\title{
Responses to Comments on Our Previous Publication (Support Care Cancer. 2018 Feb; 26(2):657-665. doi: 10.1007/s00520-017-3881-7.)
}

\section{Gary Deng ${ }^{1}$}

Received: 14 May 2019 / Accepted: 24 May 2019 / Published online: 18 June 2019

(C) Springer-Verlag GmbH Germany, part of Springer Nature 2019

\section{Comments:}

Dr. Deng and colleagues [1] presented a well-conducted study on the effectiveness of acupuncture treatment for reduction of symptom burden in multiple myeloma patients undergoing autologous hematopoietic stem cell transplantation. We have three issues which we would like to bring to light regarding this paper.

The Standards for Reporting Interventions in Clinical Trials of Acupuncture (STRICTA) were designed to improve the quality of reports on clinical trials of acupuncture [2]. In the paper by Deng and colleagues [1], no details were provided about the depth of insertion, the technique of acupuncture, the choice of acupoints whether it was unilateral or bilateral, how many acupuncture sessions in a week, and whether the patient had subjective Deqi sensation. Deqi has been proven to be of great significance in the difference of the neurophysiological analgesic mechanism between acupuncture responders and non-responders [3]. These details are very important.

In addition, the sham acupuncture group used adhesive tape to fix the needle on the surface of the skin. Patients with real and sham acupuncture may experience different sensations, which may lead to the failure of the blinding method.

Finally, the problem relates to the interpretation of the test results. If the control group was a positive drug, as long as the results would show that the acupuncture group was significantly better than the control group or had the same curative effect as the control group, it had clinical significance.

Gary Deng

dengg@mskcc.org

1 Memorial Sloan Kettering Cancer Center, 1429 First Avenue, New York, NY 10021, USA
However, if the control group is a placebo treatment, it should pre-set the clinically meaningful mean difference between the two groups. A recent randomized controlled clinical trial of pain control interventions has reported the clinically significant mean differences between the two groups ranging from 0.68 to 0.98 points [4]. That is to say this study did not make an expected estimate of the therapeutic effect. Therefore, it is difficult to guide clinicians to make qualitative judgments on the therapeutic effect.

Responses: We appreciate the interest and comments regarding our report. Here are our responses:

1. Acupuncture regimen: Description of the acupuncture regimen was abbreviated due to word limits. All acupuncture points off midline were needled bilaterally. Acupuncture needles were inserted at depth consistent with acupuncture textbooks. Deqi sensation was elicited.

2. Sham acupuncture: The patient's eyes were patched during acupuncture and they were acupuncture naïve. The technique provides minimal stimulation to the patient while maintaining satisfactory masking, as shown in Supplementary Material Online Resource 3.

3. Effect size: The study was not a fully powered phase 3 trial to determine an effect size and to guide clinical decision making. Rather, it was designed to generate an estimate of the effect size. An a priori criterion was set to "the $95 \% \mathrm{CI}$ of the reduction included a standardized difference of at least 0.5 " to deem acupuncture worthy of further study. The study results have met that criterion. A larger and fully powered study has since been planned.

Publisher's note Springer Nature remains neutral with regard to jurisdictional claims in published maps and institutional affiliations. 